\title{
Measurement of the $\Sigma$ beam asymmetry for the $\omega$ photoproduction off the proton and the neutron at the GRAAL experiment
}

\author{
V. Vegna, ${ }^{1,2,{ }^{*}}$ A. D’Angelo, ${ }^{1,2}$ O. Bartalini, ${ }^{1,2}$ V. Bellini, ${ }^{3,4}$ J.-P. Bocquet, ${ }^{5}$ M. Capogni, ${ }^{1,2, \dagger}$ L. E. Casano, ${ }^{2}$ M. Castoldi, ${ }^{6}$ \\ F. Curciarello, ${ }^{4,7}$ V. De Leo, ${ }^{4,7}$ J.-P. Didelez, ${ }^{8}$ R. Di Salvo, ${ }^{2}$ A. Fantini, ${ }^{1,2}$ D. Franco, ${ }^{1,2, \ddagger}$ G. Gervino, ${ }^{9,10}$ F. Ghio, ${ }^{11,12}$ \\ G. Giardina, ${ }^{4,7}$ B. Girolami, ${ }^{11,12}$ A. Giusa, ${ }^{3,4}$ A. Lapik, ${ }^{13}$ P. Levi Sandri, ${ }^{14}$ A. Lleres, ${ }^{5}$ F. Mammoliti, ${ }^{3,4}$ G. Mandaglio, ${ }^{4,7,15}$ \\ M. Manganaro, ${ }^{4,7,8}$ D. Moricciani, ${ }^{2}$ A. Mushkarenkov, ${ }^{13}$ V. Nedorezov, ${ }^{13}$ C. Randieri, ${ }^{3,4}$ D. Rebreyend, ${ }^{5}$ N. Rudnev, ${ }^{13}$ \\ G. Russo, ${ }^{3,4}$ C. Schaerf, ${ }^{1,2}$ M.-L. Sperduto, ${ }^{3,4}$ M.-C. Sutera, ${ }^{4}$ A. Turinge, ${ }^{13}$ and I. Zonta ${ }^{1,2}$ \\ (GRAAL Collaboration) \\ ${ }^{1}$ Dipartimento di Fisica - Università degli Studi di Roma “Tor Vergata,” via della Ricerca Scientifica 1, I-00133 Roma, Italy \\ ${ }^{2}$ INFN - Sezione di Roma "Tor Vergata," via della Ricerca Scientifica 1, I-00133 Roma, Italy \\ ${ }^{3}$ Dipartimento di Fisica - Università degli Studi di Catania, via Santa Sofia 64, I-95123 Catania, Italy \\ ${ }^{4}$ INFN - Sezione di Catania, via Santa Sofia 64, I-95123 Catania, Italy \\ ${ }^{5}$ LPSC, Universit é Joseph Fourier Grenoble 1, CNRS/IN2P3, Grenoble IPN, F-38026 Grenoble, France \\ ${ }^{6}$ Dipartimento di Fisica - Università degli Studi di Genova, via Dodecaneso 33, I-16146 Genova, Italy \\ ${ }^{7}$ Dipartimento di Fisica e di Scienze della Terra, Università di Messina, salita Sperone 31, I-98166 Messina, Italy \\ ${ }^{8}$ IN2P3, Institut de Physique Nucléaire, Rue Georges Clemenceau, F-91406 Orsay, France \\ ${ }^{9}$ Dipartimento di Fisica Sperimentale - Università degli Studi di Torino, via Pietro Giuria 1, I-10125 Torino, Italy \\ ${ }^{10}$ INFN - Sezione di Torino, via Pietro Giuria 1, I-10125 Torino, Italy \\ ${ }^{11}$ Istituto Superiore di Sanità, viale Regina Elena 299, I-00161 Roma, Italy \\ ${ }^{12}$ INFN - Sezione di Roma, piazzale Aldo Moro 2, I-00185 Roma, Italy \\ ${ }^{13}$ Institute for Nuclear Research, 60-letiya Oktyabrya prospekt 7a, 117312 Moscow, Russia \\ ${ }^{14}$ INFN - Laboratori Nazionali di Frascati, via E. Fermi 40, I-00044 Frascati, Italy \\ ${ }^{15}$ Centro Siciliano di Fisica Nucleare e Struttura della Materia, viale Andrea Doria 6, I-95125 Catania, Italy \\ (Received 30 March 2015; revised manuscript received 22 May 2015; published 25 June 2015)
}

\begin{abstract}
We report on new measurements of the beam asymmetry for $\omega$ photoproduction on protons and neutrons in hydrogen and deuterium targets from the GRAAL Collaboration. The beam asymmetry values are extracted from the reaction threshold $\left(E_{\gamma}=1.1 \mathrm{GeV}\right.$ in the free nucleon kinematics) up to $1.5 \mathrm{GeV}$ of incoming photon energy. For the first time both the radiative and the three-pion decay channels are simultaneously investigated on the free proton. Results from the two decay channels are in agreement, leading to the world's most precise measurements of the beam asymmetry for $\omega$ photoproduction off free protons. First experimental results on the deuteron allow the extraction of the $\Sigma$ beam asymmetry on quasifree nucleons. The beam asymmetry angular distributions obtained for the free and the quasifree kinematics show the same behavior, similar to the findings in pseudoscalar meson photoproduction reactions. First results of the beam asymmetry on the quasifree neutrons are presented, showing different strengths and angular distributions from the results on the proton target.
\end{abstract}

DOI: 10.1103/PhysRevC.91.065207

PACS number(s): 13.60.Le, 13.88.+e, 25.20.Lj

\section{INTRODUCTION}

The observation of nucleon excited states is fundamental for the unraveling of its internal structure. It is now understood that dynamic chiral symmetry breaking is responsible for dressing the QCD current-quark masses, which evolve into constituent quarks as their momenta decrease [1]. This creates a theoretical justification for constituent-quark models which have been used to predict the nucleon spectrum $[2,3]$.

\footnotetext{
*Present address: Physikalisches Institut - Bonn Universität, Nussallee 12, D-53115 Bonn, Deutschland; vegna@physik.uni-bonn.de

${ }^{\dagger}$ Present address: ENEA - C.R. Casaccia, via Anguillarese 301, I-00060 Roma, Italy.

${ }^{\ddagger}$ Present address: IPNL - 43, Bd du 11 Novembre 1918, Fr69622 Villeurbanne Cedex, France.

${ }^{\S}$ Present address: Insituto de Astrofísica de Canarias, E-38205 La laguna, Tenerife, Spain and Universidad de La Laguna, Dept. Astrofísica, E-38205 La Laguna, Tenerife, Spain.
}

However, important differences are still observed between the experimental nucleon spectrum and the theoretical ones; many predicted excited states have not been observed yet and have been called missing resonances.

Since the nucleon excited states decay strongly with meson emission, meson production experiments on the nucleon are the ideal environment to search for missing resonances. Most of our knowledge of the resonance properties comes from pion induced reactions. Koniuk and Isgur [4] interpreted the missing resonances as states which are weakly coupled to the pion channels and suggested to access them using an electromagnetic probe and detect final states such as $\eta N, \omega N$, or $K \Lambda$.

The study of $\omega$ photoproduction on the nucleon is interesting for several reasons. The reaction threshold lies in the third resonance region, providing access to higher mass resonances. Because of the $\omega$ meson's relatively small decay width $(\Gamma=8 \mathrm{MeV})$, its experimental invariant mass spectrum is peaked and relatively easy to distinguish from the nonresonant background. Moreover, isospin conservation 
allows only the excitation of $N^{*}\left(I=\frac{1}{2}\right)$ resonances in the reaction mechanism, while $\Delta^{*}\left(I=\frac{3}{2}\right)$ states do not contribute.

The extraction of $N^{*}$ parameters from photoproduction data would ideally proceed through experimental determination of reaction amplitudes for all spin and isospin states, using a model-independent procedure, and then relying on theoretical models to separate the resonant contributions from the nonresonant processes. In the case of vector meson photoproduction on the nucleon, 12 independent complex amplitudes are necessary to completely specify the reaction in the spin space [5], which requires measurements of at least 23 independent polarization observables, including the unpolarized differential cross section. Since the electromagnetic interaction is not isospin invariant, isoscalar and isovector amplitudes are mixed with opposite signs in the $\omega$ photoproduction on protons and neutrons. Data on both proton and neutron targets are therefore necessary to extract all reaction amplitudes from experimental results and perform a so-called "complete experiment." As this task remains very difficult for vector meson photoproduction experiments, the extraction of resonance parameters from the data relies mostly on theoretical models, which are constrained by the inclusion of new polarization observables in the database.

First measurements on $\omega$ meson photoproduction date back to the 1960s and 1970s [6-10]. Differential cross sections were measured at incoming photon energies ranging from threshold to $9.3 \mathrm{GeV}$. An exponential decay of the differential cross section with increasing values of $-t$ (square of the four-momentum transfer) was observed at the higher energies. A similar trend was also observed, at low momentum transfer, at energies close to the reaction threshold. This diffractive behavior was interpreted within the vector dominance model (VDM) as a direct $\gamma-\omega$ coupling followed by the elastic scattering of the $\omega$ meson on the proton target. More recently [11] this process has been described in terms of natural-parity exchange (Pomeron) and unnatural-parity exchange $\left(\pi^{0}\right)$ in the $t$ channel; the former dominates at high energies while the latter is the most important contribution at energies close to threshold and for small $|t|$. The role of the two contributions may be disentangled by exploiting the additional information provided by polarization observables such as the spin density matrix elements [9]. At large momentum transfer, deviations from the pure diffractive behavior are associated to $s$ - and $u$-channel contributions [10], including resonance excitation. A more direct way to estimate the contribution of intermediate resonant states consists in measuring polarization observables: in particular, the beam asymmetry $\Sigma$ is expected to be not null only if $s$ - and $u$-channel terms are involved in the $\omega$ photoproduction process.

In the past decade the study of baryon resonances has motivated several facilities to measure the $\omega$ meson differential cross section on the proton, with very high precision, in the energy region from threshold to $2.8 \mathrm{GeV}$ [12-16]. Several theoretical interpretations attempted the extraction of resonant contributions, reaching conflicting results [17-24], but all agreeing that beam polarization observables are needed to constrain the dynamics of the $\omega$ photoproduction reaction.

At present, beam polarization asymmetry has been measured only on the proton target from energy thresholds up to $1.5 \mathrm{GeV}$ in a previous analysis published by different members of the GRAAL Collaboration [15], studying the $\omega \rightarrow \pi^{+} \pi^{-} \pi^{0}$ decay channel, and up to $1.7 \mathrm{GeV}$ by the CBELSA/TAPS Collaboration [25], investigating the $\omega$ radiative decay channel. The two results are not in agreement within the quoted errors and show different angular distributions.

We are reporting on new high precision results of beam asymmetry for $\omega$ photoproduction off protons. For the first time, the $\omega$ meson is investigated both in the $\omega \rightarrow \pi^{+} \pi^{-} \pi^{0}$ decay and in the $\omega \rightarrow \pi^{0} \gamma$ radiative decay, simultaneously. We also provide the very first results of the beam asymmetry for $\omega$ photoproduction off neutrons, measured in the quasifree kinematics on a deuteron target.

\section{GRAAL EXPERIMENT}

The GRAAL experiment was located at the European Synchrotron Radiation Facility (ESRF) in Grenoble (France), where it has been taking data from 1996 to 2008 .

A linearly polarized photon beam impinged on a liquid $\mathrm{H}_{2}$ or $\mathrm{D}_{2}$ target, and the final products were detected by the large solid angle detector LAGRAN $\gamma \mathrm{E}$ (Large Acceptance GRaal-beam Apparatus for Nuclear $\gamma$ Experiments). The photon beam was produced by the Compton backscattering of low-energy polarized photons from an argon laser, against the 6.03-GeV electrons circulating inside the ESRF storage ring (see [26] for more details on backscattered photon beams).

The UV laser line was used to produce a backscattered photon beam, covering the energy range up to $1.5 \mathrm{GeV}$. By the use of the far-UV laser line, the investigated energy range was extended up to $1.55 \mathrm{GeV}$ for the reaction off free protons. A tagging system, located inside the electron ring, provided an event-by-event measurement of the photon beam energy, with a resolution of $16 \mathrm{MeV}$ [full width at half maximum (FWHM)].

Since the electron involved in the Compton scattering is ultrarelativistic, its helicity is conserved in the process at backward angles and the outgoing photon retains the polarization of the incoming laser beam $(\simeq 100 \%)$. The correlation between photon energy and polarization is calculated with QED [27] and is higher than $68 \%$ in the energy range from the reaction threshold to $1.55 \mathrm{GeV}$. During the data taking a half-wavelength plate was used to rotate the beam polarization by $90^{\circ}$ (vertical to horizontal and back) at intervals of about $20 \mathrm{~min}$, in order to collect data in the same experimental conditions with both polarization directions.

The LAGRAN $\gamma$ E detector can be divided into two angular regions:

(1) The central region $\left(25^{\circ} \leqslant \theta \leqslant 155^{\circ}\right.$ in the laboratory frame) consisting of

(i) two cylindrical multiwire proportional chambers, used for charged particle tracking, having an angular resolution of $3.5^{\circ}$ and $4^{\circ}$ for polar and azimuthal angles, respectively [28];

(ii) an inner plastic scintillator barrel, used for the discrimination between charged and neutral particles;

(iii) a bismuth germanate (BGO) electromagnetic calorimeter (see [29,30], and [31]), made of 480 
crystals and optimized for photon detection with an energy resolution of $3 \%$ at $1 \mathrm{GeV}$ and angular resolution of $6^{\circ}$ and $7^{\circ}$ (FWHM) for the polar and azimuthal angles, respectively. It has good performances also for proton detection for kinetic energies up to $400 \mathrm{MeV}$.

(2) The forward region $\left(\theta \leqslant 25^{\circ}\right.$ in the laboratory frame) consisting of

(i) two planar multiwire proportional chambers, for charged particle tracking with a resolution of $\simeq 1.5^{\circ}$ and $\simeq 2^{\circ}$ (FWHM) for polar and azimuthal angles, respectively;

(ii) a double wall of plastic scintillator bars, with a time resolution of $300 \mathrm{ps}$, for time of flight (TOF) and impact coordinates measurement of charged particles. It is used for the proton energy determination from TOF measurement;

(iii) a shower wall, with a time resolution of $600 \mathrm{ps}$ [32], for TOF and impact coordinates measurement for both charged and neutral particles. It is used for neutron/photon discrimination and for neutron energy calculation from TOF measurement. Neutral particle direction of flight may be determined with a resolution of $\simeq 3^{\circ}$ (FWHM), for both polar and azimuthal angles.

Signals from different detectors, which are found in geometrical and time coincidence, are associated and then interpreted in terms of passage of particles, according with the following considerations:

(1) In the central detector,

(i) a cluster in the BGO calorimeter, associated with a signal in the cylindrical chambers or in the barrel, is interpreted as a charged particle and its angles are provided by the tracking chambers, if present, or by the BGO.

If, within the analysis, the particle is interpreted as a candidate proton, its kinetic energy corresponds to the energy deposited in the BGO (no protons with more than $400 \mathrm{MeV}$ kinetic energy are produced in our kinematical range, for which hadronic interactions would become non-negligible); if the particle is interpreted as a candidate charged pion, it does not release all its energy in the detector and its kinetic energy is unknown.

(ii) A cluster in the BGO calorimeter, which is not in coincidence with any signal in the cylindrical chambers nor in the barrel, is interpreted as a neutral particle (photon or neutron) and its angles are provided by the location of the BGO cluster.

If, within the analysis, the signal is interpreted as a candidate photon, its energy is provided by the BGO; if it is interpreted as a candidate neutron, its energy is unknown.

(2) In the forward detector,

(i) a signal in the planar chambers in geometrical coincidence with a signal in the wall of plastic scintillators is interpreted as a charged particle.
TABLE I. List of kinematic variables directly or indirectly measured for each kind of particle.

\begin{tabular}{lcc}
\hline \hline Kind of particle & Central detector & Forward detector \\
\hline Photon & $\theta, \phi$, energy & $\theta, \phi$ \\
Neutron & $\theta, \phi$ & $\theta, \phi$, energy \\
Proton & $\theta, \phi$, energy & $\theta, \phi$, energy \\
$\pi^{+} / \pi^{-}$ & $\theta, \phi$ & $\theta, \phi$ \\
\hline \hline
\end{tabular}

If, within the analysis, the particle is interpreted as a candidate proton, its energy is calculated from the TOF, imposing the mass of the particle being equal to the theoretical value of the proton mass; if the particle is interpreted as a candidate charged pion, which is relativistic, its energy is unknown.

(ii) A signal in the shower wall, which is not in coincidence with any other signal in the planar chambers nor in the plastic scintillators wall, is interpreted as a neutral particle (photon or neutron). No energy measurement is provided for photons, while for neutrons the kinetic energy is calculated from TOF.

A summary of the kinematic variables measured by the detector for each kind of particle can be found in Table I.

At the end of the beamline, two photon flux monitoring detectors were used. The first one, with respect to the beam direction, was composed of two plastic scintillators preceded by an aluminum foil to convert photons into electron-positron pairs, while a third plastic scintillator before the aluminum foil was used as a veto for the upstream background. Its detection efficiency was low $(\simeq 3 \%$ ) to avoid pile-up effects during data taking. The second flux monitor consisted of a uniform array of plastic scintillating fibers and lead [33] (spaghetti calorimeter). Its photon detection efficiency approaches 1 and it was used to calibrate the efficiency of the former monitor, using the low intensity Bremsstrahlung beam. A detailed description of the LAGRAN $\gamma \mathrm{E}$ apparatus can be found in [34] and a schematic representation of the whole GRAAL setup is shown in Fig. 1 of the same reference.

\section{DATA ANALYSIS}

The three-pion decay $\omega \rightarrow \pi^{+} \pi^{-} \pi^{0}$ [branching ratio $($ B.R. $) \simeq 89.2 \%$ ] and the radiative decay $\omega \rightarrow \pi^{0} \gamma$ (B.R. $\simeq$ $8.3 \%$ ) are the main decay channels of the $\omega$ meson [35]. The exclusive measurement of the $\omega$ meson photoproduction on the nucleon in the radiative decay channel requires the detection of a nucleon and three photons in the final state. If the angles and kinetic energies of all particles in the final state are measured, the kinematics of the reaction is overdetermined. Since the GRAAL apparatus is optimized for photon detection and no other competing reaction has the same final state, we expect that events from this channel can be selected and separated from background. The three-pion decay channel requires the detection of two charged pions in addition to a $\pi^{0}$ and a nucleon. It is important to point out that the GRAAL detector does not provide information on charged pion energies, and that these must be deduced from kinematics constraints. In 


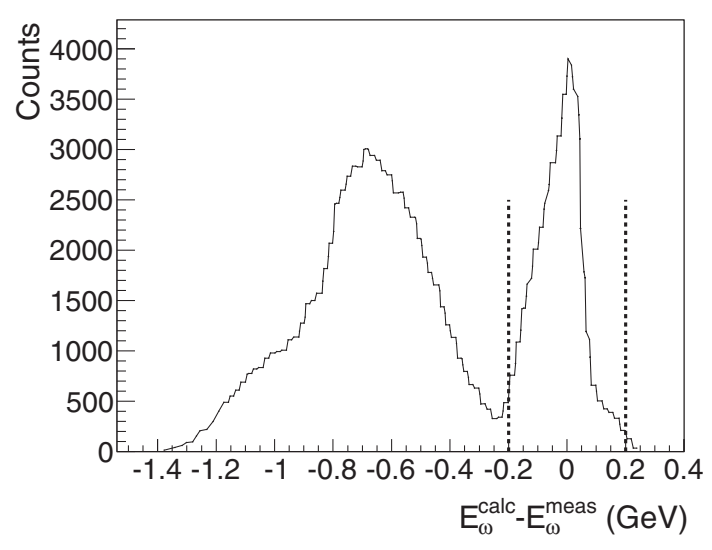

FIG. 1. Experimental distribution of the difference between the calculated and the measured energy of the $\omega$ meson. The two vertical lines indicate the range of the selected energy region (first selection cut). Simulation studies show that the background events mainly populate the bump structure corresponding to the largest difference between calculated and measured energy.

the data analysis of the free proton $\left(H_{2}\right.$ target), the target nucleon can be considered at rest and momentum conservation relations can be used to calculate the momentum strength for each of the charged pions. However, in the investigation of $\mathrm{D}_{2}$ data in the quasifree kinematics, it becomes impossible to estimate the energy (or the momentum) of the charged pions in the final state, due to the unknown Fermi momentum of the target nucleon. Moreover, the direct $\pi^{+} \pi^{-} \pi^{0}$ photoproduction cannot be separated from the $\omega$ photoproduction reaction, and it must be properly evaluated and subtracted.

Due to these considerations, the radiative and the three-pion decays have simultaneously been investigated for the free proton target only. Since the beam asymmetry measurement is independent from the $\omega$ meson decay mode, assuming that the events are integrated over the whole decay phase space, a comparison of the results obtained for the two decay channels provides a strong check on systematic errors and on their stability when different event selection techniques are used. The data analysis procedure applied for the radiative decay on the free proton is then extended to the deuteron data set, in order to study the $\omega$ photoproduction on both quasifree proton and quasifree neutron targets.

The first step in the analysis, common to all data sets and reaction channels, consists in the association of all signals from the LAGRAN $\gamma \mathrm{E}$ detector to the particle tracks, as detailed in the previous section. In the central region those tracks where at least one detector has been found in geometrical and time coincidence with the BGO calorimeter are interpreted as produced by charged particles, while neutral particles are expected to leave a signal in the BGO calorimeter only. In the forward region those tracks where at least two detectors provided a signal (either the two layers of the scintillating wall or the wire chambers and first layer of the scintillating wall) are interpreted as produced by charged particles, while neutral particles are associated to signals observed in the shower wall only.
A primary selection criterion in the analysis of all the reaction channels is the choice of a proper number of charged and neutral particle tracks in the apparatus, as explained in detail in the following subsections.

\section{A. $\omega$ photoproduction on the free proton: Radiative decay channel $\left(\omega \rightarrow \pi^{0} \gamma\right)$}

The analysis is performed for all the events showing at least one charged track and three neutral signals, among which at least two neutral particles are detected by the BGO calorimeter.

The next step consists in associating the tracks to the final state particles of the $\gamma p \rightarrow \omega p \rightarrow \pi^{0} \gamma p \rightarrow \gamma \gamma \gamma p$ reaction, without discarding events. For each charged track the missing mass of the reaction $\gamma p \rightarrow p^{\prime} X\left[M_{\text {miss }}=\right.$ $\left.\sqrt{\left(\tilde{p}_{\gamma}+\tilde{p}_{p}-\tilde{p}_{p^{\prime}}\right)^{2}}\right]$ is calculated, using the measured tagged photon energy. The charged track whose missing mass is the closest to the $\omega$ mass value $\left(M_{\omega}=782.57 \mathrm{MeV}\right.$ [35]) is interpreted as the final-state proton. The three neutral signals whose invariant mass is the closest to the $\omega$ mass value are interpreted as the three final-state photons. If one of the photons is detected in the forward direction, its energy is calculated by imposing the energy balance of the reaction. Among the three selected final-state photons, the couple whose invariant mass is the closest to the $\pi^{0}$ mass value is matched to the $\pi^{0} \rightarrow \gamma \gamma$ decay. The energy and angles of the $\omega$ meson (and of the $\pi^{0}$ from its decay) are calculated using the momentum of the three final-state photons.

Among all measured quantities, the incoming photon energy and the outgoing proton angles are the ones measured with the best resolution. Starting from these quantities and imposing two-body four-momentum conservation of the $\gamma p \rightarrow \omega p$ reaction, the proton energy and the $\omega$ meson momentum are calculated, obtaining a new set of variables $\left(E_{\omega}^{\text {calc }}, \theta_{\omega}^{\text {calc }}, \phi_{\omega}^{\text {calc }}\right.$, and $E_{P}^{\text {calc }}$ ) which can be used in the data selection procedure.

On the basis of simulation studies, it is justified to clean the data set selecting the sole events which do not show any other signals from the detector, except the ones already interpreted as the final-state proton or photons. This selection is performed requiring that no other signal appears in the forward detector, and accepting events for which a maximum energy of $5 \mathrm{MeV}$ is deposited in the BGO calorimeter by extra signals. These conditions reject background events but still save $\omega$ events for which low-energy particles are produced by the passage of the nucleon in the BGO crystals or by albedo of the detected photons.

The two remaining major background sources are identified as events from the

$$
\gamma p \rightarrow \pi^{0} \pi^{0} p \rightarrow \gamma \gamma \gamma \gamma p
$$

reaction channel, when one photon is undetected, and from the

$$
\gamma p \rightarrow \pi^{0} p \rightarrow \gamma \gamma p
$$

reaction channel, when a third random neutral signal in the detector is erroneously interpreted as the third final-state photon. Selection criteria are determined by analyzing $9 \times 10^{6}$ simulated events from all possible photoreaction channels on the proton target, in the incoming photon energy range covered by the experiment. The aim is to maximize the event selection 
efficiency and minimize the background contamination. The following selection criteria are identified:

(1) The absolute value of the difference between the measured and calculated energy of the $\omega$ meson must be smaller than $200 \mathrm{MeV}\left(\left|E_{\omega}-E_{\omega}^{\text {calc }}\right|<200 \mathrm{MeV}\right)$ (see Fig. 1, where background events are concentrated in the first bump).

(2) Since four-momentum conservation implies that the total transverse momentum of the detected particles must be null, the experimental correlation of the transverse momentum components of the reaction $P_{x}^{\mathrm{TOT}}$ vs $P_{y}^{\mathrm{TOT}}$ is calculated from the proton and $\omega$ meson trimomentum components and can be fitted using a bi-Gaussian distribution:

$$
\frac{\left(P_{x}^{\mathrm{TOT}}\right)^{2}}{\sigma_{x}^{2}}+\frac{\left(P_{y}^{\mathrm{TOT}}\right)^{2}}{\sigma_{y}^{2}} \leqslant n^{2},
$$

where $\sigma_{x}=\sigma_{y}=30 \mathrm{MeV} / c$ are the fitted widths associated to the experimental distributions of the variables $P_{x}^{\mathrm{TOT}}$ and $P_{y}^{\mathrm{TOT}}$, respectively [Fig. 2, first row, panels (a) and (b)]. The event selection is performed choosing $n=3$. This cut embeds the constraint on the co-planarity of the final-state particles.

(3) The proton missing mass must be at least $200 \mathrm{MeV}$.

(4) The invariant mass of the three final-state photons must be at least $680 \mathrm{MeV}$.

First and second cuts, shown in Figs. 1 and 2, respectively, are very effective in suppressing the two- $\pi^{0}$ photoproduction background; the third cut is needed to suppress single $\pi^{0}$ photoproduction events. The effect of the event selection constraints is monitored using the $\omega$ meson invariant mass spectrum, as shown in Fig. 3. The residual background event contamination, shown by the empty blue squared points in Fig. 3 , is estimated through the simulation to be less than $6 \%$ of all selected events.

\section{B. $\omega$ photoproduction on the free proton: Three-pion decay channel $\left(\omega \rightarrow \pi^{+} \pi^{-} \pi^{0}\right)$}

The analysis is performed for all events with at least three charged tracks and two neutral signals. The charged track corresponding to the final-state proton is identified according to the same missing mass criterion that is applied for the radiative decay (see previous section). For the $\omega$ meson reconstruction, all possible combinations of two neutral signals in the BGO calorimeter and two charged tracks in the whole detector are considered, with unknown energies and charge signs for the two candidate charged pions. Among all neutral signal pairs detected in the BGO calorimeter, the one whose invariant mass is closest to the $\pi^{0}$ mass value is selected and identified as the couple of photons from the $\pi^{0}$ decay. The selected photons are then combined with all the residual charged track couples, considered as candidate charged pions from the $\omega$ decay. As already pointed out, the energy of charged pions is not measured in the detector. Nevertheless, the charged particle track angles are known, and transverse momentum conservation law is reduced to two equations, which allows
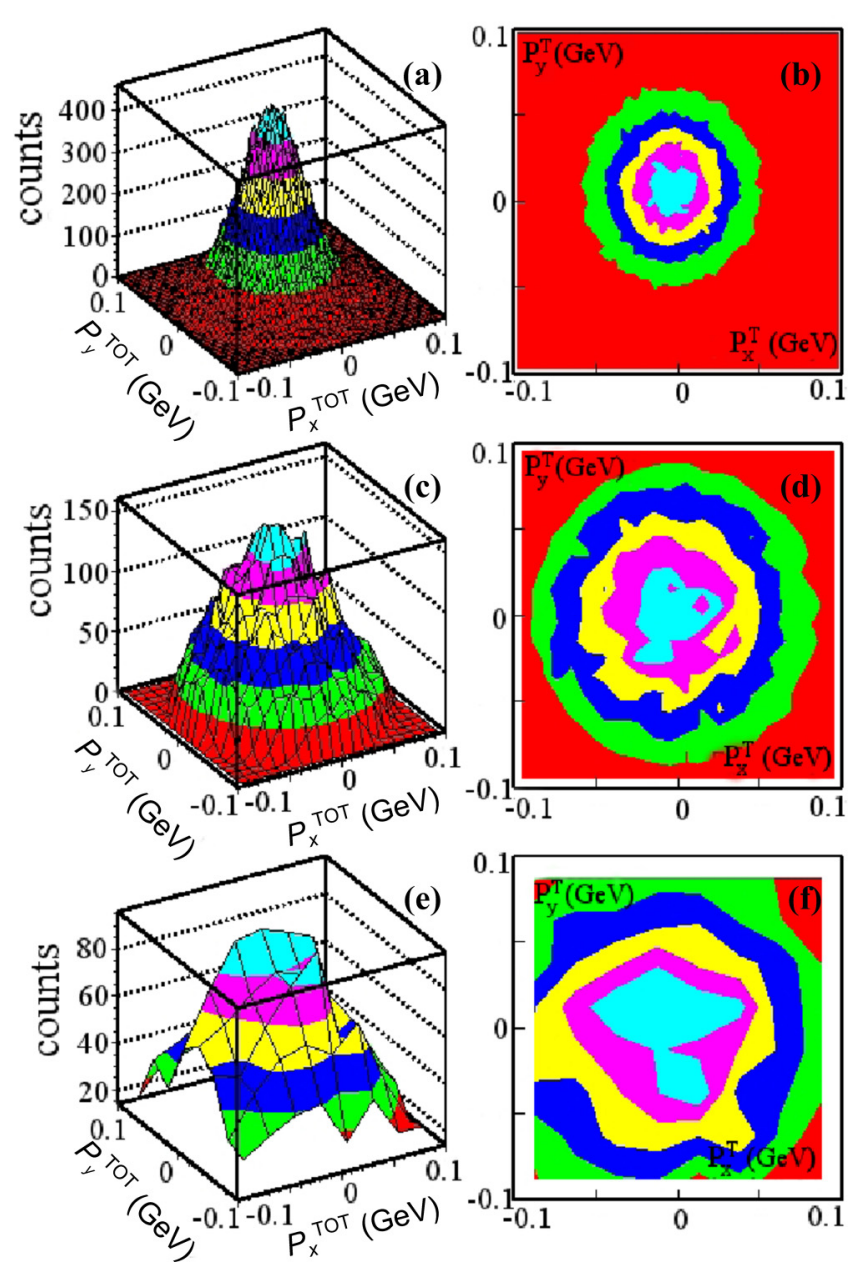

FIG. 2. (Color online) Transverse momentum distribution of final-state particles: $x$ vs $y$ distribution in a three-dimensional (3D) image (left panel) and corresponding level curves (right panel). First row, panels (a) and (b): experimental distribution on the free proton target (Sec. III A). Second row, panels (c) and (d): experimental distribution on the quasifree proton (Sec. III C). Third row, panel (e) and (f): experimental distribution on the quasifree neutron (Sec. III C).

the extraction of the two unknown pion momenta. The pair which better satisfies the longitudinal momentum conservation is selected and matched to the charged pions from the $\omega$ decay.

In order to obtain a better coverage of the phase space, the possibility that one photon from the $\pi^{0}$ decay is emitted in the forward direction is also investigated. The combinations of one neutral signal from the BGO calorimeter, one photon in the shower wall, and two charged tracks in the whole apparatus are considered. In this case not only the charged pion energies but also the energy of the forward photon is unknown. The three-momentum conservation law is used to determine the charged tracks momenta and the forward photon energy. When more than two neutral signals appear as candidate final-state photons, the pair whose $\left(\gamma_{1}, \gamma_{2}\right)$ invariant mass is closest to the $\pi^{0}$ mass is selected. All kinematical variables of the $\omega$ meson $\left(E_{\omega}, \theta_{\omega}\right.$, and $\left.\phi_{\omega}\right)$ and of the $\pi^{0}$ meson $\left(E_{\pi^{0}}, \theta_{\pi^{0}}\right.$ and $\left.\phi_{\pi^{0}}\right)$ can then be calculated. Finally, a two-body kinematics 


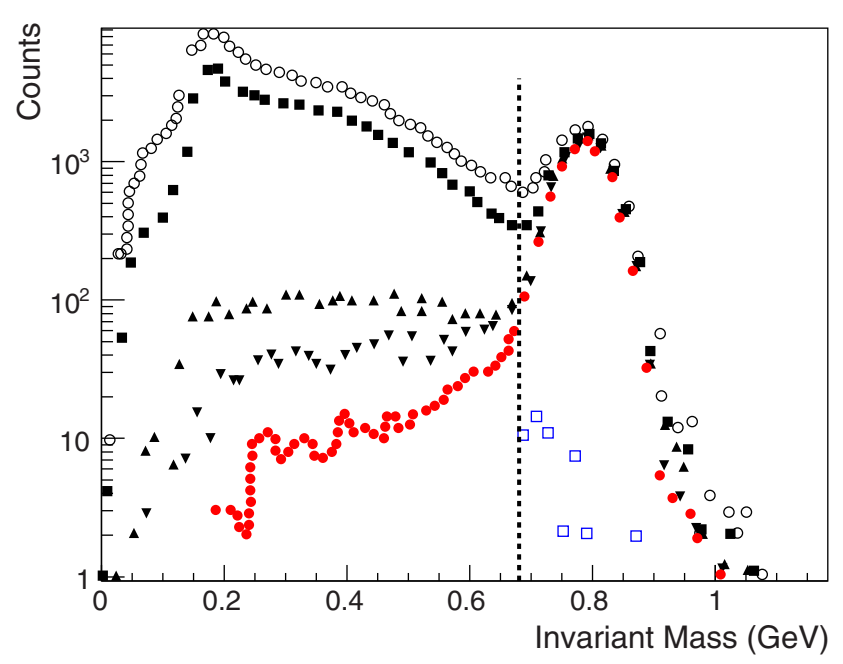

FIG. 3. (Color online) Effects of the selection criteria on the $\omega$ mass distribution for simulated data. Empty black circles: all events; full black squares: distribution after the cut on the number of residual signals in the forward detector; full black upward triangles: distribution after the cut on the energy deposited by residual signals in the BGO calorimeter (required to be smaller than $5 \mathrm{MeV}$ ); full black downward triangles: distribution after the kinematical cut on the difference between the calculated and the measured energy of the $\omega$ meson (cut number 1) and the transverse momentum conservation (cut number 2); full red circles: distribution after the cut on the proton missing mass (cut number 3 ). The black vertical line corresponds to the condition applied on the three final-state photons invariant mass (cut number 4). The distribution of the remaining background events is shown by the empty blue squares. Background contamination is estimated to be smaller than $6 \%$ of all selected events from simulation studies.

reconstruction of the $\gamma p \rightarrow \omega p$ reaction is attempted, as done previously for the radiative decay channel.

The most important difference between the radiative decay analysis and the current one lies in the event selection procedure. In the case of the three-pion decay, background events arise mainly from the direct three-pion reaction:

$$
\gamma p \rightarrow \pi^{+} \pi^{-} \pi^{0} p
$$

This represents the physical background for our process and there are no cuts allowing for background rejection, since both $\omega$ photoproduction and background events satisfy the same kinematics. Therefore, a background subtraction technique must be applied. Two cuts are previously used to clean the data set: the three-pion invariant mass must be smaller than $2 \mathrm{GeV}$ and the invariant mass of the two photons must lie in the range from $100 \mathrm{MeV}$ up to $170 \mathrm{MeV}$. Then a fitting procedure for the estimation of signal and background events is developed.

Two distributions can be used to extract the number of events: the final-state proton missing mass $\left(\gamma p \rightarrow p^{\prime} X\right)$ and the three-pion invariant mass distributions. The first one is more reliable, since it is directly calculated from measured variables (proton energy and angles). The fit of the proton missing mass distribution is performed at fixed values of the incoming photon energy $E_{\gamma}$ (4 bins), of the $\omega$ polar and

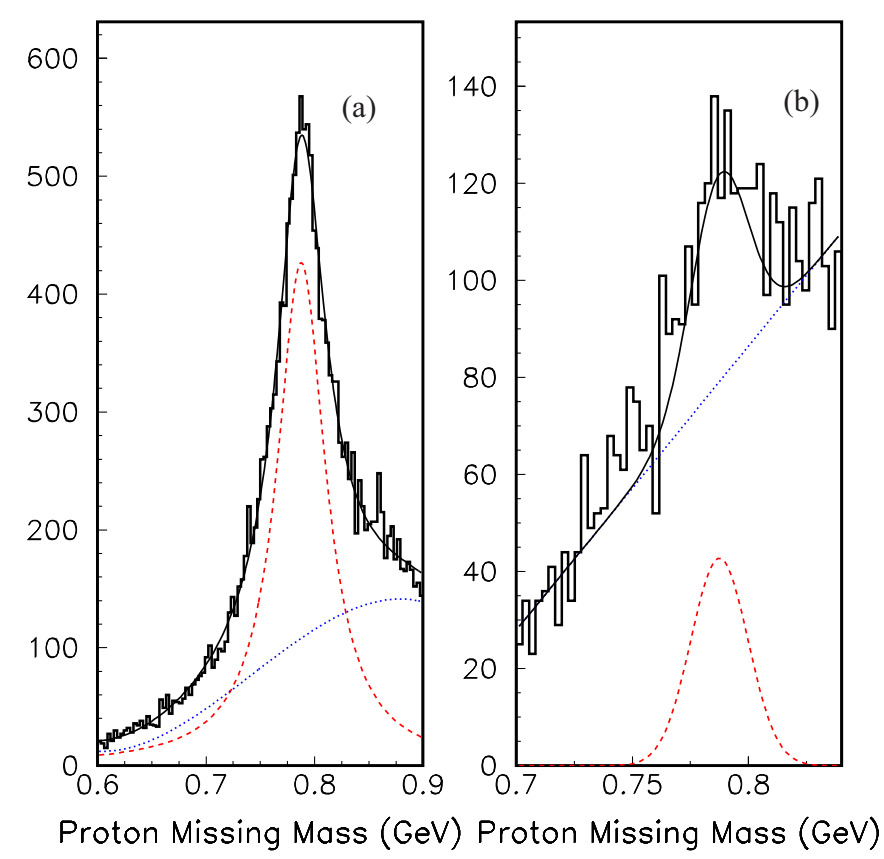

FIG. 4. (Color online) Examples of the fit on the proton missing mass distribution. Dashed line: signal contribution. Dotted line: back-ground contribution. Solid line: global fit. Panel (a) shows the experimental distribution and the fit for the events corresponding to $E_{\gamma}=1.39 \mathrm{GeV}, \theta_{\omega}^{*}=126^{\circ}, \phi_{\omega}=213.75^{\circ}$ for the vertical polarization. The $\omega$ event distribution is described by a Breit-Wiegner function while the background event distribution, mainly due to the nonresonant three-pion production, is described by a third-order polynomial function. Panel (b) shows the observed distribution and corresponding fit for the highest energy bin $E_{\gamma}=1.48 \mathrm{GeV}$, $\theta_{\omega}^{*}=162^{\circ}, \phi_{\omega}=303.75^{\circ}$ for the horizontal polarization. The $\omega$ event distribution is described by a Gaussian function while the background event distribution is described by a first-order polynomial function. In the fourth energy bin, the fitting procedure is considered not reliable due to the limited statistics and the beam asymmetry is not extracted.

azimuthal angles $\left(\theta_{\omega}^{*}\right.$ and $\left.\phi_{\omega}\right)$ in the center-of-mass frame ( 5 and 16 bins, respectively) and for each polarization state (vertical and horizontal). A total of 640 bins is fitted.

Detailed studies of simulated and experimental distributions of the proton missing mass have been performed in order to identify the best fitting function. Both Gaussian and BreitWigner distributions have been considered to reproduce the distribution of the $\omega$ events, together with several polynomial functions (first, second, and third order) to reproduce the distribution of the background events. The best fitting function has been selected on the base of the reduced $\chi^{2}$ values. It is the sum of a Breit-Wigner distribution and of a thirdorder polynomial function, for signal and background events respectively. Two examples of fitted bins are shown in Fig. 4.

The left panel of Fig. 4 [panel (a)] is shown as an example of the fitting procedure quality which has been achieved for incoming photon energies up to about $1.44 \mathrm{GeV}$. For higher energies $\left(E_{\gamma}=1.44-1.55 \mathrm{GeV}\right)$, the available statistics is much lower, as it can be deduced from Fig. 4(b), and the corresponding fits were not reliable. Due to this reason, the 
beam asymmetry for the three-pion decay channel is extracted for the first three energy bins and not in the fourth one.

\section{C. $\omega$ photoproduction on the quasifree nucleon: Radiative decay channel $\left(\omega \rightarrow \pi^{0} \gamma\right)$}

Data analysis is performed both on the proton and on the neutron target in the participant/spectator description. The reaction is thought to take place on one of the nucleons (the participant), which has a Fermi motion, while the second one (the spectator) is not involved in the process. The event selection procedure, optimized for the proton target and the $\omega$ radiative decay channel, may be extended to the deuteron data set. The final-state nucleon is identified according to the missing mass criterion. Since knowledge of the nucleon energy is necessary for the missing mass calculation, in the case of $\omega$ photoproduction on neutron target, only events with the neutron emitted in the forward direction are analyzed. The identification procedure of the three final-state photons is identical to the one performed for the free proton target.

Small changes in the analysis appear only in the selection cuts, which are optimized by analyzing $12 \times 10^{6}$ simulated events from all possible reaction channels on quasifree nucleons from a deuteron target. Because of smearing due to the Fermi momentum, the transverse momentum conservation constraint is applied with a tolerance of $4 \sigma$ (instead of $3 \sigma$ ) with $\sigma_{x}=\sigma_{y}=70 \mathrm{MeV} / c$, [see Fig. 2, second and third rows: panels (c) and (d) for the quasifree proton and panels (e) and (f) for the quasifree neutron]. An additional bidimensional selection is imposed on the correlation between the three-photon invariant mass $\left(M_{\gamma \gamma \gamma}^{\text {inv }}\right)$ and the detected nucleon missing mass $\left(M_{N}^{\text {miss }}\right)$ values:

$$
\left(\frac{M_{\gamma \gamma \gamma}^{\text {inv }}-M_{\omega}}{\sigma_{\gamma \gamma \gamma}^{\text {inv }}}\right)^{2}+\left(\frac{M_{N}^{\text {miss }}-M_{\omega}}{\sigma_{N}^{\text {miss }}}\right)^{2}<n^{2},
$$

where $M_{\omega}=782.57 \mathrm{MeV}, \quad \sigma_{\gamma \gamma \gamma}^{\text {inv }}=60 \mathrm{MeV} / c^{2}, \quad \sigma_{N}^{\text {miss }}=$ $80 \mathrm{MeV} / c^{2}$ and $n=3$. In the case of $\omega$ photoproduction on the quasifree proton, background events come not only from (1) and (2), but also from

$$
\gamma n(p) \rightarrow \rho^{-} p(p) \rightarrow \pi^{0} \pi^{-} p(p)
$$

and

$$
\gamma n(p) \rightarrow \pi^{0} \pi^{-} p(p)
$$

reactions, where the $\pi^{-}$is erroneously interpreted as a photon. These events are rejected by requiring that no signal is recorded in the plastic scintillator barrel in coincidence with a BGO cluster (not even the ones below hardware threshold in time with the event, which are included in the free-proton analysis).

In the case of $\omega$ photoproduction on the quasifree neutron, background events come from the

$$
\gamma n(p) \rightarrow \pi^{0} n(p)
$$

reaction if one photon is detected in the forward direction. In accordance with simulation studies, these background events appear mainly when the $\pi^{0}$ is emitted at backward angles and a soft photon reaches the forward region. For background events, $\pi^{0}$ and neutron must satisfy the coplanarity condition,
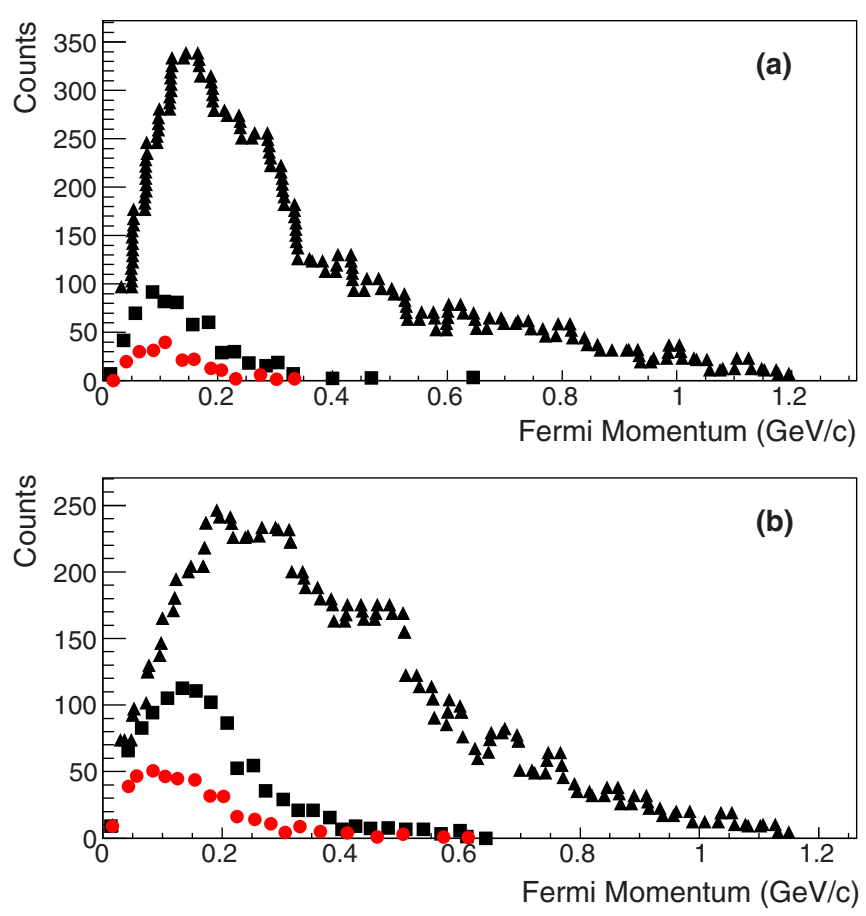

FIG. 5. (Color online) Effects of events selection criteria on the Fermi momentum distribution for the quasifree proton [panel (a)] and the quasifree neutron [panel (b)]. Fermi momentum is evaluated as described in the text. Full black triangles: Fermi momentum distributions after the cut on the transverse momentum; full black squares: distributions after the cut on the difference between the measured and the calculated energy of the $\omega$ meson, bidimensional selection on the three final-state photons invariant mass, and recoil nucleon missing mass; full red circles: Fermi momentum distributions of selected events.

which is not the case for a $\pi^{0}$ coming from the $\omega$ decay. Simulation studies of the difference $\Delta \Phi$ between the azimuthal angle of the $\pi^{0}\left(\phi_{\pi^{0}}\right)$ and of the neutron $\left(\phi_{n}\right)$ revealed that for background events $\Delta \Phi$ ranges $150^{\circ}-210^{\circ}$. In this way, events from $\pi^{0}$ photoproduction off neutron are identified and finally rejected.

The residual background events, after the described selection criteria are applied, are lower than $13 \%$ and $8 \%$ for the quasifree proton and quasifree neutron target, respectively. In Fig. 5, we show the effect of the applied selection criteria on the Fermi momentum distribution, calculated from the total momentum components of the final state $\vec{p}_{\text {TOT }}$ according to

$$
p_{F}=\sqrt{\left(p_{x}^{\mathrm{TOT}}\right)^{2}+\left(p_{y}^{\mathrm{TOT}}\right)^{2}+\left(p_{z}^{\mathrm{TOT}}-E_{\gamma}\right)^{2}}
$$

where $E_{\gamma}$ is the incoming photon energy. Final selected events show a maximum Fermi momentum value of about $400 \mathrm{MeV} / c$.

\section{Extraction of the $\Sigma$ beam asymmetry}

The differential cross section of the $\omega$ photoproduction reaction with polarized photons can be expressed in terms 


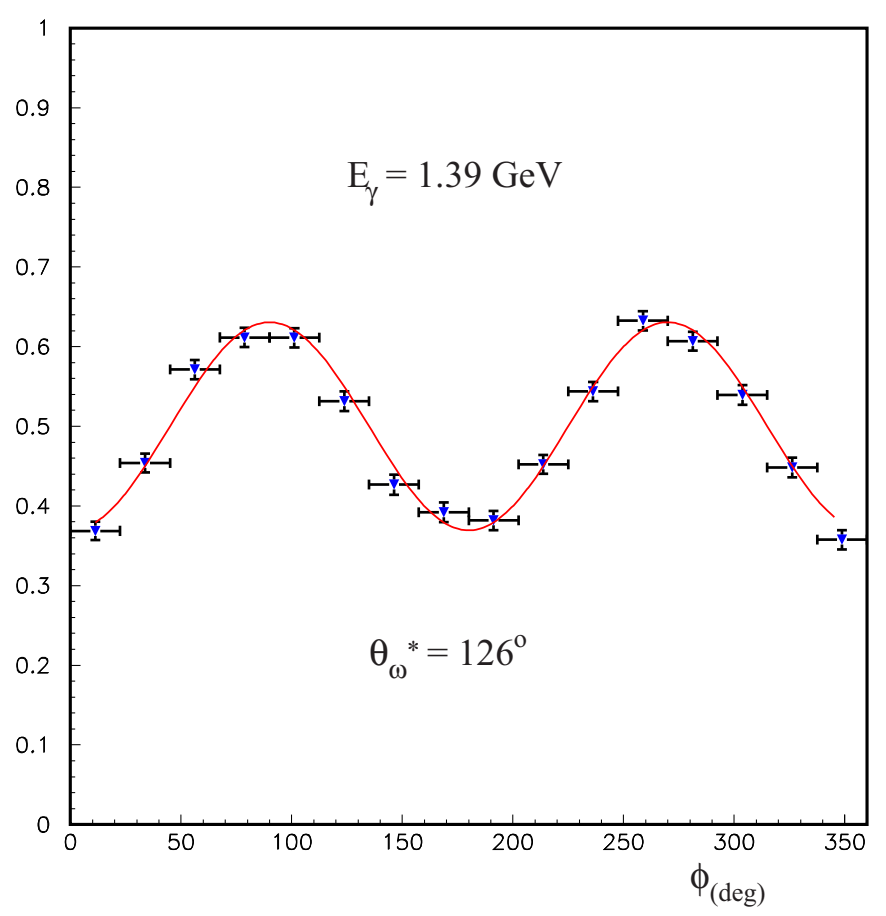

FIG. 6. (Color online) An example of the azimuthal distribution used for the extraction of the $\Sigma$ beam asymmetry at fixed values of $E_{\gamma}$ and $\theta_{\omega}^{*}$ (in this example, $E_{\gamma}=1.39 \mathrm{GeV}$ and $\theta_{\omega}^{*}=126^{\circ}$ ). The vertical error bars correspond to the statistical errors. The horizontal ones correspond to the $\varphi$-bin width.

of the unpolarized cross section as

$$
\left(\frac{d \sigma}{d \Omega}\right)_{\mathrm{pol}}=\left(\frac{d \sigma}{d \Omega}\right)_{\mathrm{unp}}\left\{1-P\left(E_{\gamma}\right) \Sigma\left(E_{\gamma}, \theta_{\omega}^{*}\right) \cos 2 \varphi\right\},
$$

where $P\left(E_{\gamma}\right)$ is the degree of polarization of the photon beam, $\Sigma\left(E_{\gamma}, \theta_{\omega}^{*}\right)$ is the beam asymmetry, and $\varphi$ is the difference between the azimuthal angle of the reaction plane $\phi$ and the incident photon polarization vector $\varphi_{\gamma}\left(\varphi=\phi-\varphi_{\gamma}\right)$. We chose to define the laboratory frame having the $\hat{z}$ component along the photon beam direction, the $\hat{y}$ component along the vertical direction, and the $\hat{x}$ component such that $\hat{z}=\hat{x} \times \hat{y}$. If the photon beam is polarized in the horizontal direction $\left(\varphi_{\gamma}=0\right)$ then $\varphi=\phi$; if the beam is polarized in the vertical direction $\left(\varphi_{\gamma}=\pi / 2\right)$ then $\varphi=\phi-\pi / 2$. In this reference frame, the polarized cross section can then be expressed as a function of the reaction plane azimuthal angle $\phi$ as follows:

$$
\left(\frac{d \sigma}{d \Omega}\right)_{H, V}=\left(\frac{d \sigma}{d \Omega}\right)_{\mathrm{unp}}\left\{1 \mp P\left(E_{\gamma}\right) \Sigma\left(E_{\gamma}, \theta_{\omega}^{*}\right) \cos 2 \phi\right\} .
$$

From the experimental point of view we can express the number $N_{H, V}\left(E_{\gamma}, \theta_{\omega}^{*}, \phi\right)$ of experimental yields, normalized by the flux of incident photons, for a given polarization as follows:

$$
\frac{N_{H, V}\left(E_{\gamma}, \theta_{\omega}^{*}, \phi\right)}{F_{H, V}\left(E_{\gamma}\right)}=d \sigma_{H, V}\left(E_{\gamma}, \theta_{\omega}^{*}\right) \Delta \Omega \varepsilon\left(E_{\gamma}, \theta_{\omega}^{*}, \phi\right) N_{\mathrm{sc}},
$$

where $F_{H, V}\left(E_{\gamma}\right)$ is the incident photon flux for horizon$\mathrm{tal} /$ vertical polarization; $d \sigma_{H, V}\left(E_{\gamma}, \theta_{\omega}^{*}\right) \Delta \Omega$ is the probability of the reaction in the $\Delta \Omega$ solid angle; $\varepsilon\left(E_{\gamma}, \theta_{\omega}^{*}, \phi\right)$ is the detection and event reconstruction efficiency, which is identical for the two polarization states; $N_{\mathrm{sc}}$ is the number of scattering centers. The $\Sigma$ beam asymmetry was extracted at fixed values of $E_{\gamma}$ and $\theta_{\omega}^{*}$ from the azimuthal distribution of the following ratio:

$$
\frac{N_{V} / F_{V}}{N_{V} / F_{V}+N_{H} / F_{H}}=\frac{1}{2}\{1+P \Sigma \cos 2 \phi\},
$$

and its value is not affected by systematic errors on the determination of the efficiency $\varepsilon$. An example of this azimuthal distribution, divided into 16 angular bins, is illustrated in Fig. 6.

\section{E. Systematics uncertainties}

The several sources of systematic uncertainties are cited in this subsection and summarized in Table II. Some of them are common to the four sets of results and do not depend on the analysis. We refer to them as to overall sources in Table II. The other ones are strictly related to the analysis procedure.

In the group of the overall sources, we consider first the uncertainties related to the determination of the photon flux and of the degree of polarization of the photon beam. These

\begin{tabular}{|c|c|c|c|c|}
\hline $\begin{array}{l}\text { Reaction } \\
\text { Decay: }\end{array}$ & $\begin{array}{l}\gamma p \rightarrow \omega p \\
\omega \rightarrow \pi^{0} \gamma\end{array}$ & $\begin{array}{c}\gamma p \rightarrow \omega p \\
\omega \rightarrow \pi^{+} \pi^{-} \pi^{0}\end{array}$ & $\begin{array}{c}\gamma p(n) \rightarrow \omega p(n) \\
\omega \rightarrow \pi^{0} \gamma\end{array}$ & $\begin{array}{c}\gamma n(p) \rightarrow \omega n(p) \\
\omega \rightarrow \pi^{0} \gamma\end{array}$ \\
\hline \multicolumn{5}{|l|}{ Overall: } \\
\hline Photon flux & $<1 \%$ & $<1 \%$ & $<1 \%$ & $<1 \%$ \\
\hline Degree of polarization & $2 \%$ & $2 \%$ & $2 \%$ & $2 \%$ \\
\hline Finite $\varphi$ binning & $<3 \%$ & $<3 \%$ & $<3 \%$ & $<5 \%$ \\
\hline Nonuniformities $[\sin (2 \varphi)$ term] & $<1 \%$ & $<1 \%$ & $<1 \%$ & $<1 \%$ \\
\hline \multicolumn{5}{|l|}{ Analysis: } \\
\hline Recoil nucleon identification & $<1 \%$ & $<1 \%$ & $<1 \%$ & $<1 \%$ \\
\hline Kinematical cuts & $<3 \%$ & $<3 \%$ & $<3 \%$ & $<5 \%$ \\
\hline Background contamination & $<6 \%$ & $<11 \%$ & $<15 \%$ & $<9 \%$ \\
\hline \multicolumn{5}{|l|}{ Total: } \\
\hline & $\simeq 8 \%$ & $\simeq 12 \%$ & $\simeq 16 \%$ & $\simeq 12 \%$ \\
\hline
\end{tabular}
determinations are common to other GRAAL analysis (as an example, to the already mentioned works [34,36-38]) and the

TABLE II. List of the sources of systematic uncertainties. 


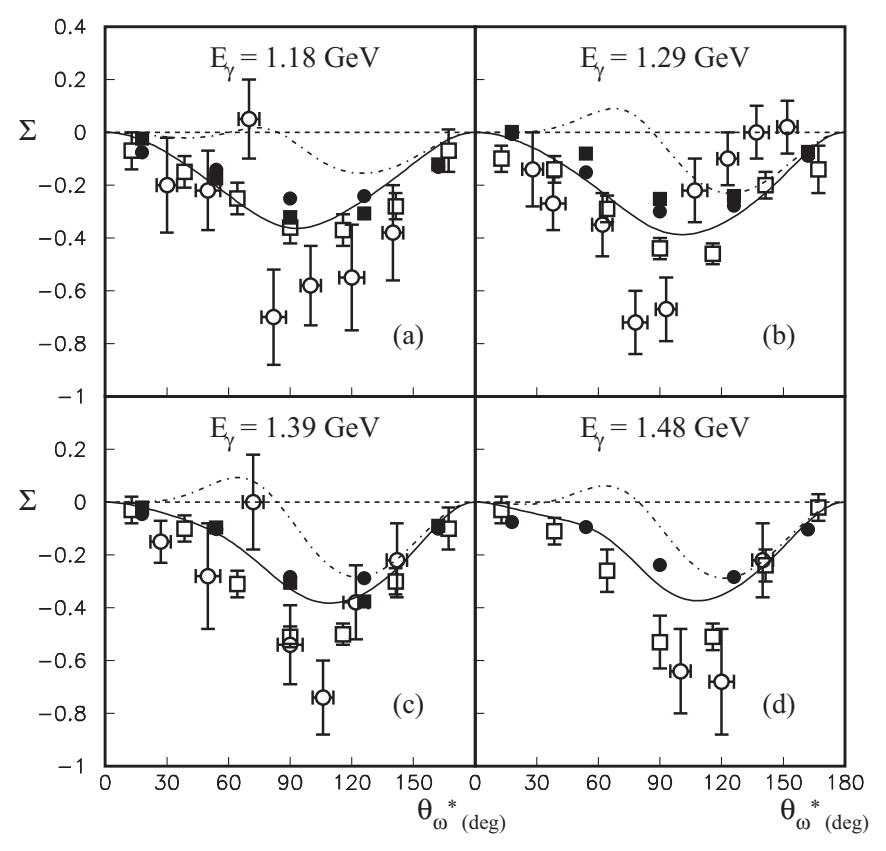

FIG. 7. $\Sigma$ beam asymmetry for the reaction $\vec{\gamma} p \rightarrow \omega p$ : both the radiative decay (full circles) and the three-pion decay (full squares) of the $\omega$ meson are investigated. Statistical errors are within the marker size. Systematical errors are not shown. Data are compared with previously published results on the three-pion decay channel [15] (open squares) and radiative decay [25] (open circles). Theoretical curves are from the model by Zhao [17,18]: solid lines correspond to the full model, dot-dashed lines do not include contributions from the $\mathrm{P}_{13}$ (1720) resonance, dashed lines correspond to the expected beam asymmetry when $s$ - and $u$-channel contributions are not taken into account.

systematic uncertainties are evaluated as smaller than $1 \%$ for the photon flux and equal to $2 \%$ for the polarization degree.

Another source of systematic uncertainties is related to the procedure for the extraction of the beam asymmetry, which requires one to bin the data in the azimuthal angle $\phi$ (as explained in Sec. IIID and shown in Fig. 6). The finite $\phi$ binning introduces a diluition of the beam asymmetry dependent on the number of bins. The data shown in Fig. 7-10 are already corrected for this effect. The correction is smaller than $3 \%$ in the case of 16 bins in $\phi$ and smaller than $5 \%$ in the case of 12 bins.

Additional systematic uncertainties due to nonuniformities of the experimental acceptance, that modify the $\omega$ finalstate particle distribution, have been investigated by adding a $\sin (2 \phi)$ term to the relation (3), which is used to fit the normalized event azimuthal distribution. No noticeable difference is found for the final results and the systematic effect is evaluated smaller than $1 \%$.

Concerning the systematic uncertainties strictly related to the analysis procedure, the first effect that we consider is a possible misidentification of the recoil nucleon. In the case of photoproduction off protons, a dedicated analysis has been performed as explained in Sec. IV A. In case of photoproduction off neutrons, we recall that the neutron is identified only in the forward part of the GRAAL detector
(Sec. II), as a signal in the shower wall in anticoincidence both with the planar chambers and the plastic scintillating wall. The probability of misinterpreting a charged particle as a neutral one corresponds to the probability of simultaneous inefficiency of both the planar chambers and the plastic scintillating wall, which is negligible. The neutron/photon disentanglement is performed on the base of the measured time-of-flight information and the systematic uncertainties on the neutron identification has been evaluated as negligible (smaller than $1 \%$ ).

For each analysis, cuts have been slightly moved around their nominal value resulting in a variation of the extracted values of the beam asymmetry smaller than $3 \%$ for the proton target and smaller than 5\% for the neutron target.

The systematic uncertainties due to background contamination have been evaluated as explained in Sec. IV B.

All the contributions have been squared and summed, resulting in a total systematic uncertainties of

$$
\begin{aligned}
& \simeq 8 \% \text { for } \gamma p \rightarrow \omega p \text {, with } \omega \rightarrow \pi^{0} \gamma, \\
& \simeq 12 \% \text { for } \gamma p \rightarrow \omega p, \text { with } \omega \rightarrow \pi^{+} \pi^{0} \pi^{-}, \\
& \simeq 16 \% \text { for } \gamma p(n) \rightarrow \omega p(n), \text { with } \omega \rightarrow \pi^{0} \gamma, \\
& \simeq 12 \% \text { for } \gamma n(p) \rightarrow \omega n(p) \text {, with } \omega \rightarrow \pi^{0} \gamma .
\end{aligned}
$$

\section{RESULTS AND DISCUSSION}

\section{A. $\omega$ photoproduction on the free proton}

$\Sigma$ beam asymmetry values are extracted for the $\vec{\gamma} p \rightarrow \omega p$ reaction from threshold up to $1.55 \mathrm{GeV}$ photon energy. The radiative and the three-pion decay channels are simultaneously investigated for the first time. Results are shown in Fig. 7 in four incoming photon energy bins as a function of the $\omega$ meson polar angle $\theta_{\omega}^{*}$ in the center-of-mass reference frame. The energy value given for each bin corresponds to the average incoming photon energy weighted by the number of corresponding events. Full circles and full squares correspond to the results from the analysis of the $\omega$ radiative decay and the three-pion decay channels, respectively. For the three-pion decay channel, beam asymmetry values have been extracted in the first three energy bins. No values are available for the fourth energy bin, due to limited statistics. Numerical values are listed in Table III.

The $\Sigma$ beam asymmetry values are negative. They are almost symmetrical with respect to $\theta_{\omega}^{*}=90^{\circ}$ in the first two energy bins and tend to be larger in the backward direction at higher energies. A very good agreement is observed between the results of the two decay channels. This comparison provides a strong check on the stability and reliability of the results since the analyses of the two channels are based on different techniques. The identification of the final-state proton is the only procedure common to both analyses. In order to verify that it does not introduce systematic errors, an alternative procedure is developed and applied to the analysis of the radiative decay. Events with more than one charged track were rejected. Protons are discriminated from charged pions using graphical cuts on the correlation plots between

(i) the energy released in the scintillating barrel and the energy deposited in the BGO calorimeter $(d E / d x$ vs energy) in the central part of the detector; 
TABLE III. $\Sigma$ beam asymmetry values for the different reactions. The $E_{\gamma}$ values (first column) correspond to the average incoming photon energy weighted by the number of corresponding photons. The $\theta_{\omega}^{*}$ values (second column) correspond to the middle value of the $\theta_{\omega}^{*}$ bins. The beam asymmetry values are listed together with their statistical errors.

\begin{tabular}{|c|c|c|c|c|c|c|}
\hline \multicolumn{2}{|c|}{ Binning } & \multicolumn{5}{|c|}{ Reaction } \\
\hline & $\overline{\left\langle\theta_{\omega}\right\rangle^{*}}$ & $\omega \rightarrow \pi^{0} \gamma$ & $\omega \rightarrow \pi^{+} \pi^{0} \pi^{-}$ & Weighted mean & $\gamma p(n) \rightarrow \omega p(n)$ & $\gamma n(p) \rightarrow \omega n(p)$ \\
\hline $1.18 \mathrm{GeV}$ & $\begin{array}{c}15^{\circ} \\
54^{\circ} \\
90^{\circ} \\
126^{\circ} \\
162^{\circ}\end{array}$ & $\begin{array}{l}-0.076 \pm 0.021 \\
-0.142 \pm 0.015 \\
-0.250 \pm 0.015 \\
-0.241 \pm 0.016 \\
-0.132 \pm 0.026\end{array}$ & $\begin{array}{l}-0.023 \pm 0.011 \\
-0.175 \pm 0.011 \\
-0.323 \pm 0.011 \\
-0.307 \pm 0.011 \\
-0.121 \pm 0.011\end{array}$ & $\begin{aligned}-0.03 & \pm 0.01 \\
-0.163 & \pm 0.009 \\
-0.297 & \pm 0.009 \\
-0.286 & \pm 0.009 \\
-0.12 & \pm 0.01\end{aligned}$ & $\begin{array}{l}-0.06 \pm 0.06 \\
-0.06 \pm 0.05 \\
-0.17 \pm 0.05 \\
-0.15 \pm 0.05 \\
-0.00 \pm 0.08\end{array}$ & $\begin{array}{r}-0.10 \pm 0.18 \\
-0.01 \pm 0.14 \\
0.09 \pm 0.09 \\
0.15 \pm 0.10 \\
-0.23 \pm 0.22\end{array}$ \\
\hline $1.29 \mathrm{GeV}$ & $\begin{array}{c}15^{\circ} \\
54^{\circ} \\
90^{\circ} \\
126^{\circ} \\
162^{\circ}\end{array}$ & $\begin{array}{l}-0.007 \pm 0.014 \\
-0.151 \pm 0.011 \\
-0.299 \pm 0.010 \\
-0.277 \pm 0.010 \\
-0.089 \pm 0.016\end{array}$ & $\begin{array}{c}0.001 \pm 0.008 \\
-0.080 \pm 0.008 \\
-0.252 \pm 0.008 \\
-0.241 \pm 0.008 \\
-0.074 \pm 0.008\end{array}$ & $\begin{array}{l}-0.001 \pm 0.007 \\
-0.105 \pm 0.006 \\
-0.270 \pm 0.006 \\
-0.255 \pm 0.006 \\
-0.077 \pm 0.007\end{array}$ & $\begin{array}{l}0.03 \pm 0.04 \\
-0.05 \pm 0.03 \\
-0.24 \pm 0.03 \\
-0.18 \pm 0.03 \\
-0.05 \pm 0.05\end{array}$ & $\begin{array}{r}-0.11 \pm 0.12 \\
-0.06 \pm 0.09 \\
0.04 \pm 0.07 \\
0.26 \pm 0.07 \\
-0.12 \pm 0.12\end{array}$ \\
\hline $1.48 \mathrm{GeV}$ & $\begin{array}{c}15^{\circ} \\
54^{\circ} \\
90^{\circ} \\
126^{\circ} \\
162^{\circ}\end{array}$ & $\begin{array}{l}-0.076 \pm 0.022 \\
-0.094 \pm 0.016 \\
-0.237 \pm 0.017 \\
-0.283 \pm 0.014 \\
-0.104 \pm 0.023\end{array}$ & & & $\begin{array}{l}-0.07 \pm 0.05 \\
-0.07 \pm 0.04 \\
-0.18 \pm 0.06 \\
-0.22 \pm 0.05 \\
-0.09 \pm 0.07\end{array}$ & $\begin{array}{l}0.15 \pm 0.16 \\
0.12 \pm 0.22 \\
0.00 \pm 0.13 \\
0.27 \pm 0.13\end{array}$ \\
\hline
\end{tabular}

(ii) the energy released and the time of flight in the scintillating wall ( $d E / d x$ vs TOF) in the forward part of the detector.

Results obtained with the new procedure are in excellent agreement with the ones from the standard analysis and we could conclude that the systematic uncertainty is well below the statistical error and we evaluated it to be smaller than $1 \%$. As a consequence, the systematics errors are determined only from the difference between the $\omega \rightarrow \pi^{0} \gamma$ results and the $\omega \rightarrow \pi^{+} \pi^{0} \pi^{-}$ones.

Three additional checks are performed both for the radiative and the three-pion decay results:

(1) Two sets of $2 \times 10^{6}$ events each are simulated by a dedicated Monte Carlo generator based on GEANT3 [39] with different input asymmetry values. In both cases the extracted beam asymmetry values are in a strong agreement with the input ones, indicating that no modification of the beam asymmetry distribution is introduced by the data analysis procedure.

(2) Beam asymmetry values are extracted by varying the $\theta_{\omega}^{*}$ and $\phi_{\omega}$ binnings. The results prove stable and independent from the binning choice.

(3) Beam asymmetry values are extracted also for background events alone, in several binning configurations. They are always compatible with zero. Therefore, if background events are erroneously included among the selected $\omega$ events, they would affect the asymmetry by reducing its magnitude. Since the two decay channels of the $\omega$ meson are analyzed with totally independent procedures, based on event selection for the radiative decay and background subtraction for the three-pion decay, and since they are characterized by different background reactions, the residual background contributions are expected to affect final results differently for the two channels. Due to the agreement of the results coming from the two decay channels (see Fig. 7), we can state that background contamination is suppressed in both channels. Since simulations have shown that background contamination is smaller than $6 \%$ for the radiative decay events, we estimate the background contamination is smaller than $10 \%$ for the three-pion decay channel.

In Fig. 7 the comparison with published data is shown: open squares are from the analysis of the GRAAL data performed by different members of the collaboration [15] for the three-pion decay channel, which do not include the tracking chambers information in the data analysis. On the contrary, this work fully exploits the good angular resolution provided to the tracking reconstruction of charged particles by the multiwire proportional chambers. While present results show strong agreement within quoted errors in the first energy bin larger asymmetry values are obtained by Ref. [15] at higher energies.

Open circles shown in Fig. 7 are from the CB-ELSA/TAPS Collaboration [25], for the $\omega$ radiative decay channel. These angular distributions, at fixed values of the incoming photon energy $E_{\gamma}$, show very different trends. The data analysis 


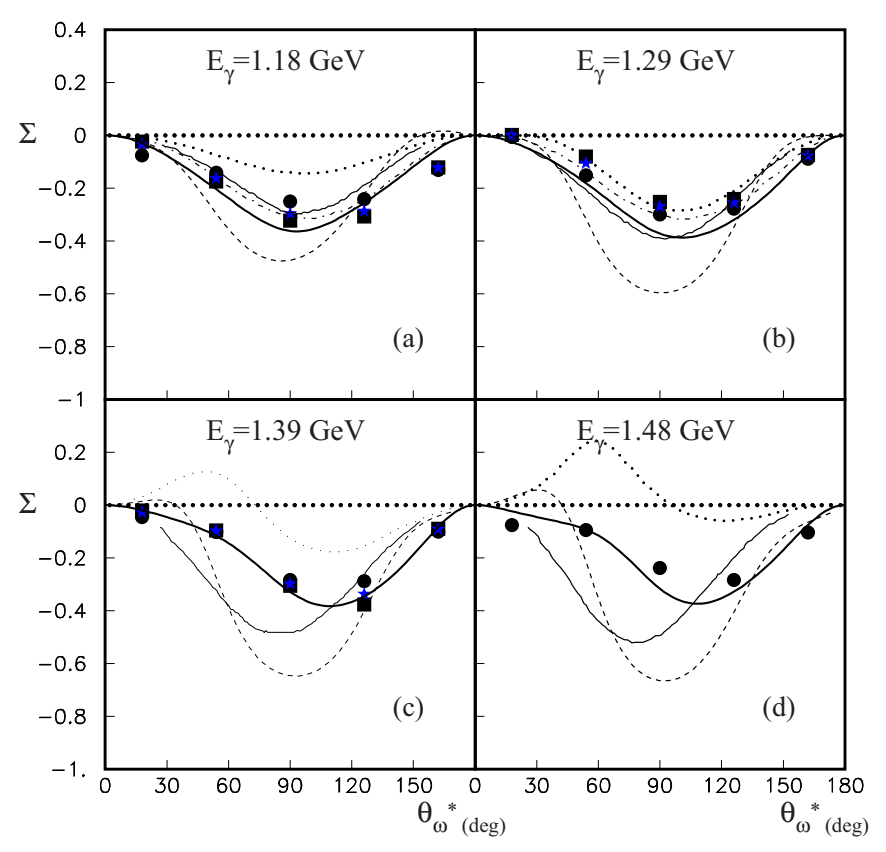

FIG. 8. (Color online) $\Sigma$ beam asymmetry for the reaction $\vec{\gamma} p \rightarrow \omega p$. Full circles are the results of the present analysis for the radiative decay channel; full squares are the results of the present analysis for the three-pion decay channel. Blue stars are the results obtained by the weighted mean of the beam asymmetry values extracted independently for the two decay modes. Statistical errors are within the marker size. Systematical errors are not shown. Thin lines are from Bonn-Gatchina partial wave analysis [22]; dot-dashed lines are the predictions from [20] at threshold; dashed lines are the results from [24]; dotted curves are predictions from Giessen model [21]; solid curves are from $[17,18]$.

from [25] is characterized by much higher background levels compared to this work, and the technique of background subtraction on the $\pi^{0} \gamma$ invariant mass spectrum is used.

The comparison of the results of this work with the previously published data shows that these are the most precise measurements of the beam asymmetry for $\omega$ photoproduction off the proton target. The results from the two decay modes have been merged through weighted mean to get a final result for $\omega$ photoproduction off proton. The averaged beam asymmetries are shown in Fig. 8 (blue stars) together with the results extracted independently for the two decay modes (full circles for the radiative decay and full squares for the three-pion decay). Numerical values for the averaged results are listed in Table III.

In the last decade, several models were developed in an attempt of determining the role and the properties of nucleon resonances from $\pi N$ and $\gamma N$ reaction data. The model in $[17,18]$ uses an effective Lagrangian approach, based on the $\mathrm{SU}(6) \times \mathrm{O}(3)$ constituent quark model, with meson-quark couplings adjusted to fit differential cross-section data. Unnatural parity exchanges $\left(\pi^{0}\right)$ and natural parity exchanges (Pomeron) in the $t$ channel are phenomenologically included. The Moorhouse selection rule [40] reduces the accessible nucleonic states from the $\left[\mathbf{7 0},{ }^{4} 8\right]$ representation, and only eight states from the lowest harmonic oscillator basis contribute to the $\omega$ photoproduction reaction in the $s$ channel. Full predictions for the $\Sigma$ beam asymmetry are shown in Figs. 7 and 8 as solid lines and are found in generally good agreement with the results of this work. The dot-dashed lines of Fig. 7 do not include the contribution from the $\mathrm{P}_{13}(1720)$, showing a large sensitivity of the polarization observable to this resonance.

An alternative model based on an effective Lagrangian approach is developed in $[19,20]$ including all $12 N^{*}$ lowest energy resonances, up to spin $J=7 / 2$, whose empirical helicity amplitudes of $\gamma N \rightarrow N^{*}$ transitions are listed by [35]. Predictions are available only close to the reaction threshold, and are shown in Fig. 8 for the two lowest energy bins as dotted-dashed lines. The model finds that the dominant contribution comes from the excitation of the $F_{15}(1680)$ state and is in fair agreement with our results.

Comparison with the Bonn-Gatchina partial wave analysis [22] is also shown in Fig. 8 as continuous thin lines. The far dominating $J=3 / 2$ wave is associated with the $\mathrm{P}_{13}(1720)$ resonance, in accordance with results from [18]. Overestimation of the $\Sigma$-beam asymmetry is obtained at the highest energies, while agreement is found closer to threshold.

A coupled-channel effective Lagrangian approach, including $\gamma N, \pi N, 2 \pi N, \eta N$, and $\omega N$ final states, is presented in [21], including all known nucleon states with spin $J=1 / 2$, $3 / 2$, and $5 / 2$ up to masses below $2 \mathrm{GeV}$. Results are shown in Fig. 8 as dotted curves. They show that the dominating contributions arise from the $\mathrm{D}_{13}$ partial wave, mainly due to the nonresonant $\pi^{0}$ exchange, and from the spin-5/2 resonances $\mathrm{D}_{15}(1675)$ and $\mathrm{F}_{15}(1680)$. Agreement with our results is visible only for the second energy bin, while a predicted change of sign at the highest energies is not observed in our results.

Another prediction comes from a dynamical coupledchannel approach, developed at the Excited Baryon Analysis Center (EBAC) [24]. Six intermediate states, including $\pi N$, $\eta N, \pi \Delta, \sigma N, \rho N$, and $\omega N$, are used to describe the unpolarized cross sections. Predictions for the $\Sigma$ beam asymmetry on the $\omega$ photoproduction data are shown in Fig. 8 as dashed lines. $t$-channel exchange contributions are not explicitly included in the calculation, and curves tend to overestimate our experimental asymmetry values.

\section{B. $\omega$ photoproduction on the quasifree nucleon}

For the first time $\Sigma$ beam asymmetry values are extracted for $\omega$ photoproduction off the quasifree proton in the $\mathrm{D}_{2}$ target, from $1.1 \mathrm{GeV}$ up to $1.5 \mathrm{GeV}$ of incoming photon energy. They are listed in Table III. Results are shown in Fig. 9 as full triangles, together with the results on the free proton (full circles) and with predictions from [17,18]. All events are obtained from the radiative decay channel analysis. An overall good agreement is found between results on the free and the bound proton, although a general trend of quasifree results to be slightly lower than free results may be noticed. As an attempt to understand the discrepancy between the free proton results and the quasifree proton ones, studies of the effects of background contamination on the extracted values of the beam asymmetry have been performed. In the hypothesis of an isotropic background (with beam asymmetry $\Sigma^{\text {bkgr }}=0$ ), 


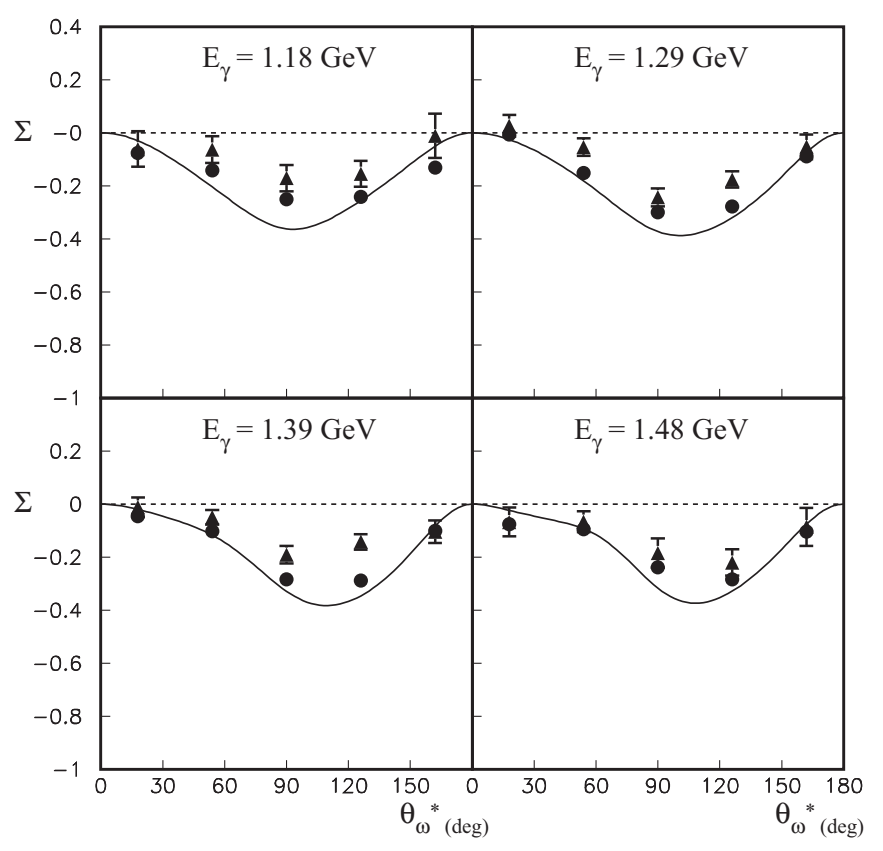

FIG. 9. Comparison of the results obtained for the $\omega$ photoproduction on the free proton (full circles) and on the quasifree proton (full triangles) in a $\mathrm{D}_{2}$ target. The error bars correspond to statistical errors. Systematical errors are not shown. A generally good agreement between free proton and quasifree proton results is observed, hinting at results for the quasifree neutron as reliable to extract information about the $\omega$ photoproduction reaction off the free neutron. Solid curves are from $[17,18]$.

the extracted beam asymmetry $\Sigma^{\text {ext }}$ would result in smaller than the pure asymmetry $\Sigma^{\omega}$ for $\omega$ photoproduction events:

$$
\Sigma^{\mathrm{ext}}=(1-f) \Sigma^{\omega},
$$

$f$ being the fraction of background events in the selected sample of data $(13 \%$, in this case). This effect results in a correction of $\simeq 15 \%$ of the values of the beam asymmetry for the quasifree proton data and it is not enough to explain the discrepancy with the free proton results.

Fermi motion effects may be responsible for the slight difference, but a dedicated theoretical investigation could be useful to better clarify the physics that lies under this small discrepancy. Nevertheless, the generally good agreement of the two results hints at similar conclusions about the reaction off the neutron. In Fig. 10 our results for the $\Sigma$ beam asymmetry for the $\omega$ photoproduction on the quasifree neutron from a $\mathrm{D}_{2}$ target are shown (empty triangles). Numerical results are also listed in Table III. These are the first results of $\Sigma$ polarization observable for the $\omega$ photoproduction on the neutron. The error bars correspond to statistical errors. Results on the quasifree neutron are compared with the ones on the quasifree proton (full triangles). The angular distribution of the beam asymmetry values differs between the neutron and proton targets, suggesting that different reaction mechanisms could be involved in the case of $\omega$ photoproduction off neutrons. For the quasifree neutron case, the beam asymmetry values are generally small and in some cases compatible with zero

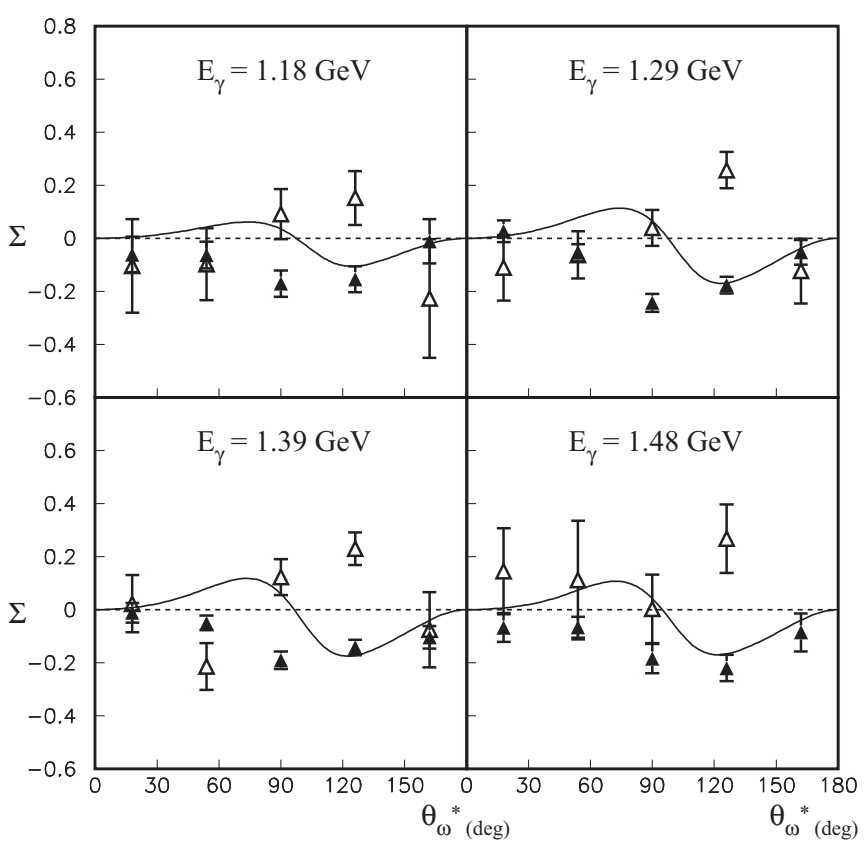

FIG. 10. Absolute first results of the $\Sigma$ beam asymmetry for the $\omega$ photoproduction on the neutron (open triangles) from the $\mathrm{D}_{2}$ target. The error bars correspond to statistical errors. Systematical errors are not shown. They are compared with the results on the quasifree proton (full triangles). Very different angular distributions are observed, indicating that different contributions are involved in the two reactions. Data are compared with the theoretical prediction from [41] (solid line) for the $\omega$ photoproduction on a free neutron target.

within the error bars. Nevertheless, the indication of a passage through zero can be observed in all energy bins at around $90^{\circ}$ (third data point), the beam asymmetry being positive at about $120^{\circ}$ (fourth data point) in all the energy bins. At present, no theoretical predictions for the beam asymmetry of the reaction $\gamma n \rightarrow \omega n$ are available in literature. In Fig. 10 our results are compared with the extension of the model [18] to the neutron case [41]. According to this model, a change of sign in the beam asymmetry values was expected at about $90^{\circ}$, but our trend looks reversed.

\section{CONCLUSIONS}

$\Sigma$ beam asymmetries are measured by the GRAAL Collaboration for the $\omega$ meson photoproduction reaction both on the $\mathrm{H}_{2}$ and the $\mathrm{D}_{2}$ targets. For the first time, results for the free proton are obtained both for the $\omega \rightarrow \pi^{0} \gamma$ and the $\omega \rightarrow \pi^{+} \pi^{-} \pi^{0}$ decay channels from the same data set and are in strong agreement. The results presented here are the most precise measurements and provide a resolution of the existing discrepancy between previously published data.

Since the asymmetry should vanish if no resonance contributes to $\omega$ photoproduction, our experimental results strongly confirm the important role of intermediate resonant states in the $\omega$ production mechanism. In particular, agreement between the experimental results and the theoretical description by [18] is observed, confirming the importance of the 
$\mathrm{P}_{13}(1720)$ resonance contribution to the reaction mechanism. Different conclusions are drawn by model [20], still in agreement with our lower energy results, but with the dominant contribution provided by the $\mathrm{F}_{15}(1680)$ resonance.

First measurements are obtained for the $\Sigma$ beam asymmetry of the $\omega$ meson photoproduction from both the quasifree proton and quasifree neutron targets in the participant/spectator analysis frame. Good agreement is found between results on the free and the quasifree proton targets, in analogy with the result found by our previous analysis for the $\pi^{0}$ and $\eta$ photoproduction reactions on the $\mathrm{H}_{2}$ and $\mathrm{D}_{2}$ targets [36-38]. A slight trend of the quasifree results to be lower than the free results may be noticed, and it may be due to Fermi momentum effects.

First results for the quasifree neutron target show that the $\Sigma$ beam asymmetry is small, compatible with zero up to $\theta_{\omega}^{*} \simeq 90^{\circ}$ but always positive at $\theta_{\omega}^{*} \simeq 120^{\circ}$. The angular distribution of the results for the neutron differs from the one measured for the proton target. This suggests that different production mechanisms contribute for the two different nucleons.

At present, no theoretical description of beam asymmetry for $\omega$ photoproduction off the neutron is available for data interpretation.

\section{ACKNOWLEDGMENTS}

We are grateful to Q. Zhao and M. Paris for the interesting and useful discussions and for the communication of most recent results. We thank the ESRF as a host institution for the stable operation of the storage ring. For the support in the maintenance of the apparatus, we thank the technical staff of the collaboration and in particular G. Nobili.
[1] M. S. Bhagwat, M. A. Pichowsky, C. D. Roberts, and P. C. Tandy, Phys. Rev. C 68, 015203 (2003); P. O. Bowman, U. M. Heller, D. B. Leinweber, M. B. Parappilly, A. G. Williams, and J. Zhang, Phys. Rev. D 71, 054507 (2005); L. Chang, Y. X. Liu, C. D. Roberts, Y. M. Shi, W. M. Sun, and H. S. Zong, Phys. Rev. C 81, 032201 (2010).

[2] S. Capstick and W. Roberts, Phys. Rev. D 49, 4570 (1994); 47, 1994 (1993); S. Capstick, ibid. 46, 2864 (1992); S. Capstick and N. Isgur, ibid. 34, 2809 (1986).

[3] D. O. Riska and G. E. Brown, Nucl. Phys. A 679, 577 (2001).

[4] R. Koniuk and N. Isgur, Phys. Rev. Lett. 44, 845 (1980).

[5] M. Pichowsky, C. Savkli, and F. Tabakin, Phys. Rev. C 53, 593 (1996).

[6] BHMTW Bubble Chamber Group, Phys. Rev. 155, 1468 (1967).

[7] ABBHHM Collaboration, Phys. Rev. 175, 1669 (1968).

[8] Y. Eisenberg, B. Haber, Z. Carmel, E. Peleg, E. E. Ronat, A. Shapira, G. Vishinsky, R. Yaari, and G. Yekutieli, Phys. Rev. Lett. 22, 669 (1969).

[9] J. Ballam et al., Phys. Rev. D 7, 3150 (1973).

[10] R. W. Clift, J. B. Dainton, E. Gabathuler, L. S. Littenberg, R. Marshall, S. E. Rock, J. C. Thompson, D. L. Ward, and G. B. Brookes, Phys. Lett. B 72, 144 (1977).

[11] B. Friman and M. Soyer, Nucl. Phys. A 600, 477 (1996).

[12] F. J. Klein et al., $\pi N$ Newsletter 14, 141 (1998).

[13] J. Barth et al., Eur. Phys. J. A 18, 117 (2003).

[14] M. Battaglieri et al., Phys. Rev. Lett. 90, 022002 (2003).

[15] J. Ajaka et al., Phys. Rev. Lett. 96, 132003 (2006).

[16] M. Williams et al., Phys. Rev. C 80, 065208 (2009); 80, 065209 (2009).

[17] Q. Zhao, Z. Li, and C. Bennhold, Phys. Rev. C 58, 2393 (1998).

[18] Q. Zhao, Nucl. Phys. A 675, 217c (2000); Phys. Rev. C 63, 025203 (2001).

[19] Y. Oh, A. I. Titov, and T.-S. H. Lee, Phys. Rev. C 63, 025201 (2001).

[20] A. I. Titov and T.-S. H. Lee, Phys. Rev. C 66, 015204 (2002).
[21] V. Shklyar, H. Lenske, U. Mosel, and G. Penner, Phys. Rev. C 71, 055206 (2005); G. Penner and U. Mosel, ibid. 66, 055211 (2002); 66, 055212 (2002).

[22] A. V. Anisovich, A. Sarantsev, O. Bartholomy, E. Klepmt, V. A. Nikonov, and U. Thoma, Eur. Phys. J. A 25, 427 (2005).

[23] M. Paris, Phys. Rev. C 79, 025208 (2009).

[24] A. Matsuyama, T. Sato, and T.-S. H. Lee, Phys. Rep. 439, 193 (2007).

[25] F. Klein et al., Phys. Rev. D 78, 117101 (2008).

[26] D. Babusci, V. Bellini, M. Capogni, L. Casano, A. D’Angelo, F. Ghio, B. Girolami, L. Hu, D. Moricciani, and C. Schaerf, Riv. Nuovo Cimento 19, 1 (1996).

[27] D. Babusci, G. Giordano, and G. Matone, Phys. Lett. B 355, 1 (1995).

[28] A. Lleres et al., Eur. Phys. J. A 31, 79 (2007).

[29] P. Levi Sandri et al., Nucl. Instrum. Methods Phys. Res., Sect. A 370, 396 (1996).

[30] M. Castoldi et al., Nucl. Instrum. Methods Phys. Res., Sect. A 403, 22 (1998).

[31] F. Ghio et al., Nucl. Instrum. Methods Phys. Res., Sect. A 404, 71 (1998).

[32] V. Kouznetsov et al., Nucl. Instrum. Methods Phys. Res., Sect. A 487, 396 (2002).

[33] V. Bellini et al., Nucl. Instrum. Methods Phys. Res., Sect. A 386, 254 (1997).

[34] O. Bartalini et al., Eur. Phys. J. A 26, 399 (2005).

[35] K. A. Olive et al. (Particle Data Group), Chin. Phys. C 38, 090001 (2014).

[36] R. Di Salvo et al., Eur. Phys. J. A 42, 151 (2009).

[37] O. Bartalini et al., Eur. Phys. J. A 33, 169 (2007).

[38] A. Fantini et al., Phys. Rev. C 78, 015203 (2008).

[39] GEANT, Detector Description and Simulation Tool. CERN program Library Long Writeup.

[40] R. G. Moorhouse, Phys. Rev. Lett. 16, 772 (1966).

[41] Q. Zhao (private communication). 